\title{
Similar Examinations, Different Tests: A Comparative Description of the SAT AND JUKEN SYSTEMS
}

\author{
Ian Roth ${ }^{\mathrm{a}}$ \\ Meijo University
}

\begin{abstract}
This paper compares the SAT and juken. It does this at three levels of analysis: structure, function, and participant communications. While the prior two rely on publicly available information and established theories, the latter is based on the analysis of data collected from social media. The findings of this paper are that, while the two examinations are structurally and functionally similar enough to appear ready points of comparison, their differences are profound enough to make such comparisons misleading. Among the consequential differences discussed are that, as opposed to the SAT, the juken is a longer, more consequential process that is more likely to impose dependency upon its participants. In combination, these differences result in a higher-pressure process that challenges its participants not only intellectually, but socio-culturally, and characterologically. As a result, this paper suggests that, whereas the SAT serves a functional role in determining university admissions in the US, the juken is a central facet of Japan's institutionalized education and socialization process.
\end{abstract}

Keywords: entrance examinations, jukensei, educational systems, university admissions, SAT

\section{Introduction}

Around the world, variations on university entrance examinations are used during or following the final year of secondary education as summative assessments of students' academic abilities. The various examinations are structured in different ways, but their core function is to serve as sorting mechanisms identifying which tertiary institutions students have a chance of entering, and which students those institutions should be considering. Given their consequence, then, it is unsurprising that the preparations undertaken for examinations of this kind are more expensive, intensive, and extensive than those associated with any examinations encountered earlier in the schooling process.

The Scholastic Aptitude Test (SAT) and juken are both examples of such pre-tertiary sorting examinations-consequential standardized tests taken by students seeking to attend university in the United States and Japan respectively. The SAT is the most common such instrument in the US, though others such as the ACT (not an acronym) are also widely used. Juken, which translates to 'entrance examination', is the collective name for any of the examinations used in determining school eligibility. This includes examinations required by schools ranging from the primary to tertiary levels. But, the capstone of this entrance examination system is the university juken which, again, is a term that encapsulates any number of different examinations used to determine university eligibility. It is this iteration of the juken with which this paper concerns itself and to which the term juken will hereafter refer.

While the SAT and juken share elements of structure and function-both are university gatekeeping, standardized assessments of academic ability-they also differ in both dimensions.

a Correspondence can be directed to: iroth@ccalumni.meijo-u.ac.jp 
And, this paper argues, neither can be properly understood without a third dimension: how those participating in the examinations communicate about their related experiences.

This paper provides structural and functional comparisons of these e before investigating how the experience of preparing for them translates into a spontaneous, peer-to-peer conversation conducted over social media. The questions it seeks to answer are

1) How are the two examinations structurally and functionally similar and different in ways that are likely to impact the experiences of those preparing to take them?

2) In what ways does this population communicate amongst itself about their shared preparation experiences?

3) How closely aligned are these structural-functional elements and the intra-group communications they produce and what does this suggest about the experience of preparing for either?

\section{Three Lenses: Structure, Function, and Communication}

The analytical method employed here is an assessment of the examinations through three lenses, each intended to reveal a particular layer of operations. This method was adapted from Banathy (1995). These lenses will be referred to as structure, function, and communication. The structure lens asks the questions, "What is there and how is it organized?". The function lens asks, "What services does it perform? What are its inputs and outputs?". The communications lens asks, "What do those participating in the system communicate to one another about that system?". In combination, these lenses produce a thorough description of these examinations.

\section{Structural Comparison: The First Lens Similarities}

Both the SAT and juken are first taken during a student's final year of high school in order to satisfy university entrance requirements. While taking the SAT post-high school is entirely permissible and not unheard of, it is also not as institutionalized an option as the post-high school juken. Undoubtedly this is because, whereas the SAT was offered seven times during the 2018-2019 academic year (CollegeBoard, 2019), the juken was offered a single time: it is an annual event. The differences in frequency are interdependent with differences in how pre-examination testing is conducted. For the SAT, students are likely to take informal practice tests often timing and correcting for themselves or having their SAT teacher/tutor perform these duties. Jukensei, or students preparing for the juken, also do these things. But, in their case, mock examinations are conducted by cram schools and universities throughout the year leading up to the examination. These are highly formal, all-day affairs, nearly indistinguishable from the real juken. Participants are provided with detailed score reports and their national ranking for each subject they took.

The SAT consists of three required sections: reading, writing and language, and mathematics, which contains no-calculator and calculator-permitted sub-sections. There is also an optional essay component which, when taken, lengthens by fifty minutes the normally three-hour long test (Compare SAT Specifications, n.d.). By contrast the Center Test-the first round of the juken that is honored by a majority of universities - is spread out over two days, takes up to eleven hours, and requires participants to take anywhere from six to nine different examinations in subjects ranging from world history, to classical Japanese, to Physics, to a foreign language (NCUEE, 2015). Examination-takers choose the subjects they take but, often, their choices are dictated by the university departments they are attempting to enter.

This relates to another major difference between the two examinations. While they are both examinations employed in common by a range of universities, the SAT experience does not vary depending on which university a person is attempting to enter. The Center Test portion of the juken 
as taken by two different people may cover very different subjects. Whereas SAT scores are often used by students to calibrate the level of university they should be applying to, takers of the Center Test must have already identified a limited collection of university departments that they both believe they can qualify for and that share testing requirements. A student who does not qualify for their chosen departments may be left with only the options of trying again a year later or of entering a lower-level university that does not require entrance examination scores. The need to have already calibrated which departments are within reach is only functional thanks to the prevalence of the mock examination system.

Assuming a jukensei does well enough on the Center Test, he or she may have to then take an institution-proprietary, department-specific examination. For such students, the juken is essentially an examinaiton tournament in which a sub-par performance at any stage is disqualifying (Takeuchi, 1997).

The cost involved in preparing for and taking the respective examinations is also a point of dissimilarity. The cost of the SAT with the essay portion is under seventy dollars. Other costs include those associated with SAT prep courses or tutoring which, though optional, are commonly employed by those who can afford them. Since 2015, the CollegeBoard and Khan Academy have collectively offered a free, online SAT prep (CollegeBoard, 2016). Preparing for the juken almost always requires attending some manner of cram school, often for a year or more. Most of the mock examinations are organized by these private institutions and cost as much as the SAT (Kawaijuku, 2019). And the examinations that comprise the juken, particularly the institution-specific ones, are significantly more expensive. Though the newest version of the SAT is designed to reflect the Common Core curriculum, spending money on extra preparation is warranted for those who can afford it because neither country's standard high school curriculum fulfills the demands of its entrance examination (Mori, 2002; Mulvey, 2001). As a result, success on either examination requires targeted, often expensive, preparation.

The SAT is meant to serve as one among several consequential factors used in determining university acceptance. With over 1,000 US universities-prestigious institutions among themhaving dropped standardized testing as an admission requirement, however, this is no longer a given (FairTest, 2019). High school transcripts have traditionally received equal consideration, and research suggests high school grade point average (GPA) predicts both first-year and fourth-yearcumulative university GPA as well as the latest version of the SAT (Montoya and Camara, 2012). Extracurricular activities, particularly athletics, are considered and, in the case of certain universities and certain applicants, can be the determining factor. Even the application itself, with its essays, letters of recommendation, and attendant interview, can be enough to identify an applicant as qualified for entrance.

Efforts have begun to more seriously consider the non-juken qualifications of those applying to Japanese universities. But, they are either in their infancy-as in the case of International Baccalaureate (IB) recipients (Yamamoto, Ishikura and Saito, 2016)—or, since most Japanese institutions "...essentially live a hand-to-mouth existence covering current operating expenses and capital expenditures primarily from student fees" (Kinmouth, 2005, p. 108), are best understood as maneuvers intended to support healthy enrollment numbers rather than evaluative integrity (McCrostie, 2017; Mori 2002). Essentially, the juken is still considered the most reliable path into Japan's selective institutions of higher education (Aspinall, 2003; Zeng, 1999).

While the juken consists, for many students, of multiple rounds including institution-specific examinations, the first of these rounds is often the Center Test which is similar to the SAT in that it is a common examination employed by many institutions. The former is designed and administered by an Independent Administrative Institution tied to the government while the latter is a product of an non-profit organization named the College Board. 
Table 1: Summary of Structural Comparison

\begin{tabular}{|c|c|c|}
\hline & SAT & Juken \\
\hline Timing & Prior to entering tertiary education & Prior to entering tertiary education \\
\hline Style & Standardized, largely multiple choice & Standardized, largely multiple choice \\
\hline Subjects covered & $\begin{array}{l}\text { Reading, writing and language, } \\
\text { mathematics, and optional essay }\end{array}$ & $\begin{array}{l}\text { Japanese, Physics, Foreign languages, } \\
\text { Geography, Japanese History, World } \\
\text { History, etc. (Students take a selection } \\
\text { based on requirements of desired } \\
\text { university's and departments }\end{array}$ \\
\hline Frequency & Offered seven times a year & Offered once a year \\
\hline Preparation & $\begin{array}{l}\text { Preparation involves informal } \\
\text { practice tests }\end{array}$ & $\begin{array}{l}\text { Preparation involves informal practice } \\
\text { tests and formal mock examinations }\end{array}$ \\
\hline Cost & $\begin{array}{l}\text { Under } 70 \text { US dollars USD) with a } \\
\text { waiver program for low income } \\
\text { students; often supplemented with } \\
\text { prep classes and/or tutoring }\end{array}$ & $\begin{array}{l}\text { Taking three or more subjects on the } \\
\text { Center Test costs 18,000 Japanese } \\
\text { Yen (JPY) (approximately } 180 \text { USD), } \\
\text { while two subjects costs } 12,000 \text { ( } 120 \\
\text { USD); University specific examinations } \\
\text { cost extra ( } 35,000 \text { JPY for Tokyo City } \\
\text { University) as do each round of Mock } \\
\text { Examinations (between } 5,000 \text { and } \\
7,000 \text { JPY) }\end{array}$ \\
\hline $\begin{array}{l}\text { Weight in university } \\
\text { admissions }\end{array}$ & One of several weighty factors & Nearly the only determining factor \\
\hline
\end{tabular}

\section{Function: The Second Lens}

Both examinations function as sorting mechanisms, ranking students by score according to their demonstrated academic ability. While what exactly constitutes 'academic ability' is itself a contentious issue, the examinations share a focus on knowledge retention and retrieval, on problem solving through the application of concepts, on reading and writing proficiency, on conscientiousness as a character trait, and on the ability to perform in prolonged, intellectually demanding, highpressure situations. Less explicitly, the examinations also sort for resourcefulness and for the power and reliability of a student's support network.

Though these aspects of academic ability are targeted by both examinations, among the most consequential differences in function between them is simply the extent to which they challenge 
students to perform in these dimensions. Many of the elements contributing to the challengethe length of the preparation period, the length and difficulty of the examinations, the associated costs, and the weight of those examinations in determining university eligibility-were covered in the previous section. But, seeing that it is as much a functional aspect of the examinations as a structural one, the final of these deserves more extensive exploration here.

Both examinations perform a sorting function, identifying appropriate matches in a way that serves both students and institutions of tertiary education. But, whereas the function of the SAT is to contribute to that sorting, the juken essentially determines it. A proper understanding of why this is the case requires tying together the institutional diversity and rigor exhibited by universities with the recruiting methods employed by businesses.

While there are academic leaders among US universities, many institutions that are less than outstanding overall are nevertheless recognized as outstanding in certain disciplines. The diversity of strengths and institutional character among US universities contribute to higher education in that country functioning as a market with a comparatively wide variety of both supply and demand. The lack of a similar diversity among Japanese universities, however, disproportionately strengthens the position of the top institutions to select from among the highest scoring applicants. This is true to the extent that students commonly choose to attend the highest ranked university that accepts them, without regard for the department in which this will place them (Mori, 2002). This has contributed to a weakening of the positions of many less selective institutions to the point that some have collapsed and many more are on the verge of doing so (McNeill, 2008).

Central to this dynamic is Japan's national university rankings being based on the juken performances of the students they enroll. The result is a reinforcing loop wherein a university's attractiveness to students-its ranking - is determined by its ability to attract those students. The motive power behind university rankings is more than merely the prestige they promise. A second reinforcing loop exists in which a university's name and ranking attract students who are then able to form reciprocal networks with one another that will be of relied upon post-university when the members are likely to find employment in a variety of organizations. While orthogonal to the quality of education on offer, the networking possibilities implied by a university's rank provide justification for the value it purports to deliver, further feeding into the institution's name value and the validity of the entire ranking system (Murphy, 2014). Each time an organization hires without concern for what an applicant studied, instead basing their decisions on the rank of his or her university and the networks he or she is assumed to have cultivated, the nascent importance of those university networks and of the score and rank focused decisions of students and universities is further validated (Takeuchi, 1997).

Further enforcing the importance of the juken and standardization of universities are the implicit limitations on academic rigor that they must accept. For a US university, rigor is an adjustable dimension of the institution's character. Largely owing to the need to ensure sufficient enrollment, failing poorly performing students is not a common practice for Japanese universities. Securing entrance is largely viewed as the difficult part of university; after this, students can rely on the, "... unspoken rule that, by accepting students, an institution has a duty to graduate them," (Goodman 2003 , p. 23). As a result, for universities to maintain the strength of their reputations as based upon the graduates they produce, the initial hurdle - the juken-must be made the most challenging one (Zeng, 1999). 


\section{Figure 1: The Juken Feedback Loops}

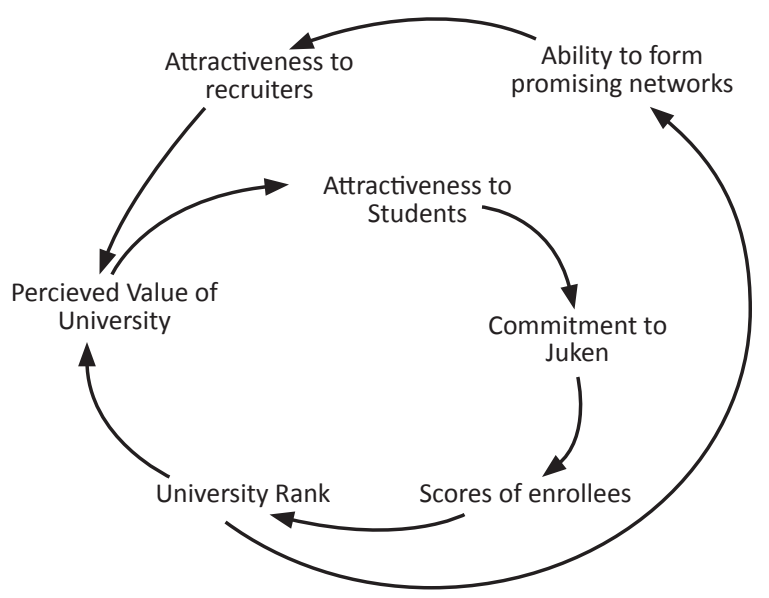

Each actor is acting rationally - students choose the university that offers the most promising future, universities choose the students that will best impact their rankings, buffering their financial solubility by easing the path to graduation, and recruiters hire the graduates most likely to arrive with a beneficial network in tow. The result is a series of reinforcing dynamics driving the system to recognize juken scores as the sole variable determining a causal chain that extends far beyond being admitted to university. The effects of this on the concerned actors are that the consequences associated with the juken become extreme (Tsukada, 1991) and the diversity present in the higher education ecosystem is purged. Therefore, while US universities display a variety of profiles, the products on offer in the Japanese higher education market are diverse in reputation, mixed in quality, and standardized in kind. In contrast to the US "mountain range" style system, the Minister of Education, Culture, Sports, Science, and Technology described Japan's higher education landscape as a "Mt. Fuji system" with the University of Tokyo residing at the summit (FPCJ, 2018).

With respect to those taking the examinations, and on account of the factors just explored, the systems perform deeply different functions. It is possible to do well on the SAT despite an underwhelming academic record. And it is possible to subsequently excel in a respectable university to the point of revivifying one's future possibilities. With the SAT acting as a gatekeeper, university in the US is a kind of institutionalized reset beyond which what came before ceases to be pertinent. The juken, the educational system it serves, and the culture that informs them, are more grounded. The determination of an individual's future probabilities begins from the earliest stages of education (Arai, 2016). The juken is the culmination of years of formal and informal schooling, of family and economic background, of friendships, and of the entire preceding education and socialization process. It is where the adolescent demonstrates his or her fitness according to the relevant cultural demarcations. "...the competition in the entrance examination is their competition for increased self-esteem to prove their potential or worth..." (Tsukada 1991, p. 102) In this way, the distinction between SAT and juken preparation can be summarized as follows: whereas contending with the SAT is one of the pre-requisites for engaging in the initiatory rite of passage that is university in the US (Puglia, 2016), contending with the juken is the entirety of Japan's initiatory rite of passage (McVeigh, 2000; Zeng, 1999, Tsuda, 1993).

Considering the juken as performing the socio-cultural and psychological functions of an initiatory rite of passage leads to the question of what outcomes are associated with the examination 
understood in this way. The first of these is the reinforcement of the ganbaru ethic which is characterized as persistent, maximal effort and endurance despite hardships. Given the extent to which the rest of Japan's education system seems designed to instill a ganbaru mentality (Murphy, 2014; Sheftall, 2011; Zeng, 1999; DeVos, 1973), it is predictable that the final, cumulative challenge of that system rewards extreme capacity in this regard (Aspinall, 2005).

A second clear socializing function of the juken process is the reinforcing of ties of obligation, particularly those connected to family members. The ganbaru mentality's centrality in Japanese culture is rivaled by that of dependency on others and the webs of obligation it produces. These are at the root of Murphy's observation that, "....ordinary Japanese people take their responsibilities seriously" (2014, p. 254). As opposed to the Western concept of original sin, the core motivating concept of the Japanese religio-cultural system is something akin to original debt: "The conviction that one has already received blessings, benefits, and favors from one's superiors... is the terminus a quo of moral feeling, the basic presupposition of ethical discourse and conduct" (Davis 1992, p. 19).

The demands of the juken foist upon the participant a significant, additional chunk of such debt. And those unwilling or not in a position to take on such debt are unlikely to succeed, at least to the degree of entering a highly selective university. With regard to the ganbaru mentality, the same combination of ability and willingness is required. Those who do not fulfill any single combination present on the resulting matrix-able/willing to ganbaru/be dependent-are thereby filtered out of the traditional leadership track, and those who remain are more thoroughly socialized to enact precisely these patterns of persistent self-application and human network-dependency (Zeng 1999, p. 2).

The final point to be made in this regard is the degree to which jukensei seem to accept the system and its processes. In combination with the rigidly rank-ordered hierarchy of the universities, the power of the juken itself functions as a socializing instrument for, unlike in the US, university hopefuls desirous of entry into a selective institution have little recourse but to accept the orthodoxy. By so doing, they both individually submit to being further socialized in accord with its demands and re-legitimize it as a social institution (Tsukada, 1991). As a result, the students themselves, beyond the complaints and minor rebellions that intensive and extensive examination preparation may inspire, often accept the system at a deep level (Tsukada, 1991). As an instrument, the juken is well-suited to its context-its requirements are merely those of its containing socio-cultural system magnified. To summarize, the juken understood as an initiatory rite of passage rewards the ability and willingness to ganbaru, to take on obligation, and to accept the status quo thereby strengthening committed self-application as a character trait, reinforcing the individual's social ties through feelings of indebtedness, and ensuring his or her suitability as a soon-to-be member of society.

Before setting aside the function lens, a final differentiating point must be addressed. That point is the role played by the examinations as mechanisms of the university entrance system with respect to the larger system in which it is embedded. The multi-factor admission process in the US takes into account consequential interviews, admission essays, and the consideration of potentially impactful extra-curricular resumes-all of which require interpretive evaluation on the part of admission boards. This ensures that the process is self-evidently subjective. Applicants to US universities do not assume their admission will be determined entirely by algorithm. Central to the juken's purported utility, however, and to public tolerance for it despite its associated costs, is its perceived ability to function as an objective instrument (Guest, 2006; Zeng, 1999; Tsukada, 1991). The consequences of university admissions are serious enough, and the tendency of the society towards particularism strong enough, that an objective means of apportioning university spots provides a necessary counterbalance (Aspinall, 2005; Zeng, 1999; Rohlen, 1983). Public acceptance of the juken's objectivity, despite evidence to the contrary, is a lynchpin that holds together the education system's veneer of meritocracy (Zeng, 1999). Thus, it functions symbolically and pragmatically both at the level of individual experience and of public perception. 


\section{Communication: The Third Lens}

The daily experience of contending with these examinations is no less a product of interactions between those contending than of the relevant structures and functions. The third lens will focus on this aspect of the process.

\section{Materials and Methods}

The data used in this section comes from the social media platforms of Twitter and Instagram. Data such as this provides several advantages beyond its quantity and public availability. Most pertinent for the purposes of this paper are that it is spontaneously produced and not elicited in any way by the researcher making it, in this way, more authentic than data collected through contrived means.

As a data source, social media posts are still relatively new. Nevertheless, they have been recognized as useful for both quantitative and qualitative research being, in the case of the latter, compared with person-to-person surveys (Social Media Research Group, 2016). This report goes on to identify thematic analysis as an appropriate methodology for dealing with social media data. It cautions that, on account of the performativity associated with the medium "...both positive and negative feelings are over-stated..." (Social Media Research Group 2016, p. 14).

Examples of researchers who have used posts such as those employed here include Turkle (2011), Shanahan, Brennan, and House (2019), and Ozawa-de Silva (2008). Shanahan, Brennan, and House (2019) collected posts related to self-harm from Twitter, Instagram, and Tumblr, drawing conclusions based solely on analyses of the images they contained. My own prior research suggests that the sentiments expressed in social media posts align closely with those expressed during interviews (Roth, 2019). And, perhaps the most consequential point justifying social media posts as a data source is that even in the edge case of every post being somehow disingenuous, online communications are fundamentally of consequence as they affect those exposed to them. Research conducted by Hogue and Mills, for example, suggests that exposure to "...attractive peers' appearance-based social media resulted in worsened body image..." $(2018$, p. 3). Thus, it can be extrapolated that how students engage with the examinations in question here could be similarly influenced by their exposure to examination preparation-related posts. These examinations are no less collective experiences than they are individual ones. Understood as a system, one's peers are just as much elements of these examinations as are the materials used to study or the examinations themselves. The difference, of course, is that peers are purposeful elements and social media provides a means of studying their purpose-driven behaviors

\section{Data Collection}

In the case of the juken, posts made on these services by jukensei were collected daily throughout the month of November, 2017. In the end, over 700 posts were collected. Similarly, English-language posts related to preparing for the SAT that were made on either of these services over the last five years were collected. This yielded approximately 70 posts.

Given the nature of the platforms, such collection efforts can never be provably exhaustive. In both cases, the most common tags used to characterize posts as related to either the juken or the SAT were identified and searched for. These tags were \#浪人生＃受験生，\#浪人生ライフ，\#受 験生と繋がりたい,\#ろうにんせいと繋がりたい (respectively translated as \#roninsei ${ }^{1}$,\#jukensei, \#roninseilife, \#wanttoconnectwithjukensei, and \#wanttoconnectwithroninsei for the juken and \#SAT, and \#SATprep for the SAT. All posts made and so tagged within the designated time period were collected. These tags were chosen because they were the most frequently used tags that could reasonably be assumed to identify posts related to the research topic. Collecting every post related to the topics in question would have been impossible, but the tag search-based collection method 
allowed a representative sample of what each group said regarding the preparation process to be assembled.

\section{Data Treatment}

The collected posts were then subjected to a three stage, thematically-focused Hermeneutic Content Analysis (HCA-T) following a slightly modified version of the process detailed by Vieira and de Queiroz (2017, p. 12-14). Once the research topic and data to be used had been identified process, this consisted of constructing a coding framework with defined thematic categories, of testing that framework with sample sets drawn randomly from the complete data sets, of evaluating and modifying the coding framework, and of conducting the main coding. This process was performed for each data set.

In order to ensure the coding process was sufficiently reliable, I re-coded approximately twenty percent of the data from the jukensei set and one hundred percent of the SAT group data following a two-week period during which I had no exposure to the data. The two sets of coding were then compared with the following results.

Table 2: Comparison of Original Coding and Re-coding

\begin{tabular}{|l|l|l|l|l|l|}
\hline Data Set & $\begin{array}{l}\text { Themes } \\
\text { Coded } \\
\text { During Initial } \\
\text { Coding }\end{array}$ & $\begin{array}{l}\text { Themes } \\
\text { Missed } \\
\text { During Re- } \\
\text { coding }\end{array}$ & $\begin{array}{l}\text { Percentage of } \\
\text { Themes Missed }\end{array}$ & $\begin{array}{l}\text { Themes } \\
\text { Added During } \\
\text { Re-coding }\end{array}$ & $\begin{array}{l}\text { Percentage of } \\
\text { Themes Added }\end{array}$ \\
\hline Jukensei & 180 & 11 & $6.1 \%$ & 21 & $12.4 \%$ \\
\hline SAT & 147 & 10 & $6.8 \%$ & 18 & $12.2 \%$ \\
\hline
\end{tabular}

These results suggest that the coding categories were defined with sufficient clarity. They also suggest that the initial coding process was more likely to have underestimated rather than overestimated the thematic complexity of the data. Thus, the coding results appear to be reliable if not exhaustive. In the service of transparency, the results section contains numerous examples of how utterances were coded.

The modifications made to Vieira and de Queiroz's process were that, due to the quantity of data and broad range of themes represented therein, the coding frameworks went through several iterations before they become completely serviceable. Data such as images were also coded, though only according to what was objectively present (e.g. study materials, food). In that this was a comparative analysis, the exhaustive coding of each data set was followed by a cross-data set comparison that focused on identifying points of convergence and divergence, and gaps. In order to close the hermeneutic circle, the broad and comparative analyses of the data sets were followed by a delving into of each theme that had been identified in order to extract discrete examples of how these themes manifested themselves. The goal was to identify the utterances which provided the most direct access to the essence of each theme. Many of these are presented in the results section.

\section{Results}

This section will present the results of the coding before examining those themes identified as consequential by the comparative analysis. To support claims made about these themes, social media posts made by individuals preparing for the SAT or juken will be quoted. The associated examination is indicated prior to each of these quotes and the codes assigned to them are presented in brackets 
following them. Quotes about the juken have been translated from Japanese by the author. The findings will be presented in three parts: themes that converge, themes that diverge, and themes with only unilateral representation, referred to as gaps.

\section{Coding Results}

The jukensei data was broken into sections of approximately 30 posts. Thus, the corresponding table (see Appendix A) includes data about the rate of appearance of themes in sections as opposed to in the entire data set. This should be understood to be the percentage chance that the theme in question appears at least once in a random selection of 30 or so posts. This information can be used to identify how widespread a theme was as opposed to how densely expressed. The SAT group data (see Appendix B) includes the rate of appearance for each theme in the entire data set.

\section{Convergent Themes}

The first convergent theme is an overwhelming focus on the negatively-valenced experiences associated with preparation. This is not to suggest that the process is represented as entirely unpleasant, but that both the quantity and potency of negative experiences are portrayed as outstripping those of positive experiences.

[SAT] this is the "super tired" face of mine \#lifesohard \#SaturdayMorning \#endofsemester \#holiday \#SAT \#SATPractice all bullshit [Valence-Negative; Complaint; Schedule]

[Juken] I don't know what I've been doing this whole time [....] when I finished calculating my score I was honestly dumbfounded. On the way home, I saw some people who looked like university students. They had such happy expressions on their faces. I asked myself what it is that I am even doing, and I felt sad. [Valence-Negative; Progress-Results; Being JukenseiCharacterizations]

Among jukensei, negative opinions were voiced more strongly and approximately twice as often as positive ones. Among the SAT students, however, the ratio was close to three to one negative to positive and, with rare exception, the positive utterances related to peripheral elements of the SAT experience such as friends. More specifically, much of the convergent negativity expressed related to feelings of disappointment regarding perceived progress and anxiety about the upcoming examinations.

[SAT] Another day, another practice test but yet my Reading and Writing and Language score stays within the same range [Valence-Negative; Ability]

[Juken] I only feel anxious. It's bad. What can I do to pass the Center Test...? Compared with last year, I understand more things but, for some reason, my scores aren't going up. [ValenceNegative; Examinations-Center; Progress-Ability and Difficulty; Progress-Results]

At the same time, both groups also expressed anticipation for the break they would enjoy once finished with the examinations.

[SAT] Looking foward [sic] to spring break, much needed rest [Valence-Positive; Break]

[Juken] Recently, as the examinations get closer, I feel more and more scared. Sometimes I'm not able to sleep. But, today I thought about how, once I have passed, I can go out to eat and do lots of other fun things with my friends. I realized that now is my only opportunity 
to challenge myself and I need to work hard. [Valence-Positive/Negative; Examinations-Post; Progress-Motivation; Leisure]

Social media provided an avenue for both groups to behave boastfully and humbly with respect to their abilities.

[SAT] I never opened this thing [SAT Prep book], but I got a really high score on my English portion of the SAT (: [Valence-Positive; Ability; Materials]

[Juken] My Center test scores this time were so good. I wonder if other people are making this much progress. They went up so much I don't completely believe they are correct. [ValencePositive; Examinations-Center; Progress-Results]

[SAT] in math i use this thing called the guess and hope method [Ability]

[Juken] My Japanese ability is very bad. I answered some questions yesterday for the first time in a month and I couldn't answer them at all. [Valence-Negative; Progress-Ability and Difficulty]

It was common for both groups to post the results of their mock or practice examinations. But, whereas the SAT group typically posted scores as a single picture containing one percentile rating accompanied by a short reaction, the jukensei posted multiple detailed score sheets and often went on at length comparing each of those scores to their previous performances.

Both groups recognized the primacy of conscientious action in achieving their examination goals. They posted similar pictures of organized desks accompanied by proactive utterances.

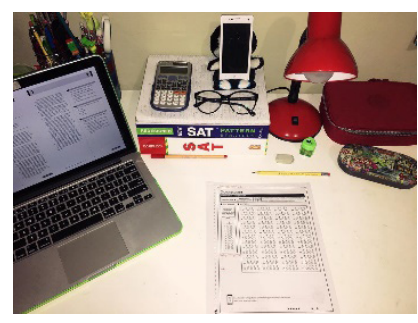

[SAT] [Materials]

Practice! Practice! Practice! [Effort-Intention]

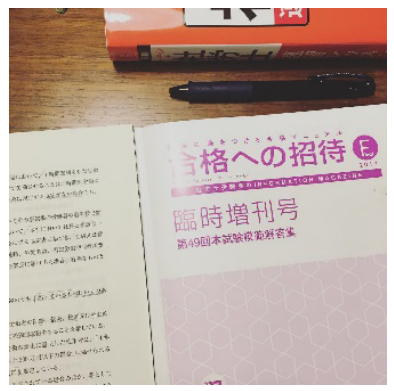

[Juken] [Materials]

Just get it done! [Effort- Intention] 
[SAT] Welp, I'm officially back to the grind.... I've been studying for the SAT all morning and figuring out college admissions! Already missing Christmas!! 周 [Valence-Negative; Effort-Prior]

[Juken] My reflection on this week: I studied for 55 hours. Recently, I haven't been able to plan when I'll have time to study[...] I sometimes can't even find three hours to study. [ValenceNegative; Preparation-Time Studying; Effort-Prior]

The number of posts focused on time spent was offset by a smaller number acknowledging that hours put in did not necessitate progress made-that the productive quality of that time was also a necessary consideration.

[SAT] So not focused tonight :/ ugh [Valence-Negative; Effort-Prior]

[Juken] During this 8 hours and 40 minutes, I only studied for two hours. I can't even remember how I spent the rest of the time. Ahhhh... what a waste. I spent six or seven hours by myself just thinking about random things. [Valence-Negative; Preparation-Time studying; ConditionMental; Effort-Lack]

The social media services were also used as means to request and offer support. The nature of this support varied, from preparation material related to strategic and psychological in nature.

[SAT] guys does anybody have any of the previous Official SAT full tests Pdf, I need them urgently. [Support; Materials]

[Juken] Knowledge Grade-Up Seminar is of a different quality than the other lectures I have used. It's more stimulating [...] it will help you connect principles and application in the way you think. [Being jukensei-Advice about; Preparation-Materials]

[SAT] Legit upset at how un Marvelous my grade is. \#SATprep \#Tutor? One that will take their time with me to translate how to solve the problems and tips to solve them. So I can maybe master this. 边 [Valence-Negative; Ability; Support]

[Juken] My heart feels like it's going to break, but looking at everyone's posts I feel like I can keep trying as well. I'm not just saying that. I feel like I can do it. [Valence-Negative; SupportFollowers; Progress-Ability and Difficulty]

A final point of thematic convergence is portrayal of food and drink as sources of both support and reward that could be integrated into the preparation efforts.

$[\mathrm{SAT}]$ Milked coffee + Kaplan + Music $=$ the best mood for studying =)) [Valence-Positive; Food and Drink]

[Juken] These are Lamune [a candy] that my mom gave me before. They were hiding in my bag. So I'm going to eat them. When you are tired... Sugar! And Chocolate! [Valence-Positive; Food and Drink; Condition]

[Juken] I rewarded myself for my hard work with this new Starbucks dessert. [Valence-Positive; Food and Drink] 


\section{Divergent Themes}

The themes to be discussed in this section will be those that are present in some form in both data sets. Whereas the convergent themes were those that contained consistent sentiments expressed about similar aspects of the preparation experience, the divergent themes are those that, while portraying similar experiential aspects, proved inconsistent across sets.

Both groups posted pictures of themselves but, whereas the SAT group did so seemingly without concern, the pictures from the juken group almost never included any unobscured faces. Posters would often take the extra step of positioning a sticker or blurry spot in order to obfuscate their faces.

Also with respect to how social media was used, there is a staggering disparity in the level of engagement demonstrated by the two groups. Data collection for this study turned up approximately 70 posts made over the last five years by SAT students about the examination and preparation for it. Over 700 posts were made by jukensei in a single month. Considering the relative sizes of the SAT student and jukensei populations, this disparity in engagement is even greater than it appears.

There is also divergence in how the SAT and juken groups deride their respective examinations and complain, or refrain from complaining, about the preparation that it requires.

[SAT] Dear @CollegeBoard I hate you more than I hate Eggplant Thank you :) [ValenceNegative; Complaint]

[SAT] Literally never felt less like Friday in my life [Valence-Negative; Complaint; Schedule]

[SAT] Why wake up early to study when im [sic] already in summer.[sic] [Valence-Negative; Complaint; Schedule]

[Juken] The time we have to deal with any remaining problem questions from the Center Test has gone from 60 days to 50 days. I know that from now I must go turbo speed. I really need to treat each and every day as important. [Examinations-Time left before; Preparation-Time studying; Examinations-Center]

[Juken] Why do I need to do this? Even if I do it, how will it be useful in the future? Math? Will I use it? History? What's the point? Classical Japanese? Am I going to use that? I feel like laughing about it. But, you can't only do the things you want to do. Do what you want when you can, but also do what you must even when you don't want to. The worst thing is to not act. Those who don't act never have a chance to succeed. They won't have the life they want. So, when you run into a wall and are uncertain the only thing to do is face yourself, get over it, and take confident action. [Being jukensei-Advice; Reflection-Last Year or Earlier]

These quotes demonstrate two points of difference in how the groups portray their experiences. First, members of the SAT group appeal to a sense of unfairness about the examination, suggesting grudging acknowledgement that preparation is necessary by studying despite the burden they claim it is placing on their lives. In comparison, jukensei refrain from maligning the examination (the above quotation was the closest thing to a complaint in the entire data set). Rather, they express recognition that such negativity would harm only them, that the burden is of-their-own-choosing, and that resolute self-application is the only reliable way to forestall future regrets.

The second distinction on display in the above quotes is how the groups portrayed their preparation time. The SAT students suggested this time was akin to an unfortunate casualty or a nearly unwilling sacrifice. Jukensei, however, portrayed their preparation time as a scarce and valuable resource. The overall impression is that, whereas members of the SAT group suggested a feeling of deprivation associated with forfeiting to preparation what could have been an enjoyable night or a lazy morning, the jukensei bemoaned never having enough such nights or mornings to so invest. They represented examination preparation as a race against time rather than as an imposition on it. 
Consistent with this are the divergent ways in which the two groups express their feelings about time spent studying. For the SAT group, the clearest sentiment expressed was annoyance at the time required to prepare. For the jukensei, it was pride and regret. They regularly boasted about the amount of time they had spent studying, posting pictures of timers showing the number of hours they had studied that day. There were also instances of jukensei self-organizing to create accountability measures. One cohort competed based on hours studied, reporting the top five for each week. Here is an example of one of these posts with the usernames changed:

[Juken] To begin with, I have collected last week's data. Here are the results:

First- @fdhao_fios 92h33m

Second- @yydduu 92h28m

Third- @ $\mathrm{bbb}$ $78 \mathrm{~h}$

Fourth- @PO_yu 72h53m

Fifth- @suim 64h [Support-Followers; Preparation-Time Studying/Routines and Systems]

Regret was expressed when posters felt as though they had not been able to dedicate enough time to studying. In such cases, a sense of having missed an opportunity or of falling behind was typically expressed.

\section{Thematic Gaps}

The following themes are those which were present in one of the data sets, but for which a converging or diverging correlate could not be found in the other data set.

Jukensei represented their test preparation experience in a way the SAT did not: as one fundamentally characterized by a process of self-overcoming.

[Juken] Even though I can say without hesitation that I know more than I did last year, my score hasn't changed. It's because I am weak. When I'm not concentrating, I blame it on all sorts of things. That is why I probably won't develop. I'm scared. Really. But, I won't fail. Because I must pass. Because I want to pass more than anything. [...] What happens around me isn't important. It's myself that I can't lose to. [Valence-Negative; Reflection-Last year or earlier; Progress-Results/Goals; Effort-Lack]

Considering the relationship to time that jukensei develop, it is unsurprising that they also described struggles with health issues in a way that the SAT students did not. Jukensei commiserated over headaches and sleeplessness; they offered and reported heeding advice about dealing with sickness.

[Juken] I don't know how long this has been going on, but I haven't been getting good sleep. I close my eyes and just can't fall asleep. It's so bad that, when I saw my mom on Sunday, she said my eyes seemed lifeless. What do I need to do to get to sleep? Is this because I've accumulated a lot of stress? It's already November, so I'm just going to attack with everything and give up on any kind of withdrawal. [Valence-Negative; Condition-Mental/Physical; EffortIntention] 
[Juken] I feel like there are a lot who think they can just go on being more and more sleep deprived until they take the exam, but that is totally not true. Making your body weak during winter, when you have all this stress, living an unhealthy lifestyle, not getting enough exercise-these things add up and will beat you. You don't want your physical health to ruin all this hard work you've done. [Being jukensei-Advice about; Condition-Physical]

The jukensei also spoke about the upcoming examinations in much starker, more evocative terms than did the SAT group. Many of them portrayed the juken as a life-defining challenge-as something to be approached in a thoroughly resolved manner or not at all.

[Juken] The battle is won or lost starting now. The problems I face will not control my future. Those who honestly face their problems and keep working even while crying are also able to find enjoyment in the toughest of times!! I choose to do what I should do. [Effort-Intention]

[Juken] To think that such a wonderful, idyllic, respected university is waiting for me [...] If I need to, I will give my last breath for that. I will not give up until the very end! [UniversityLife/Of interest; Progress-Goals; Effort-Intention]

[Juken] With regard to what is most serious: so that spring will see the blooming of cherry blossoms, in the face of difficulties you must stand up and walk forward. [Effort-Intention; Being jukensei- Characterizations]

These posters balance tragic language with powerfully positive words and symbols. Such descriptions suggest that the jukensei experience is one characterized by an existential gravity. No such language was employed anywhere in the SAT data group. Rather, the experience was portrayed as being inconvenient but necessary, annoying though temporary and, overall, as an unfortunate event.

The themes of obligation and gratitude constituted another gap. While SAT preparation courses and tutors may cost money, they are more likely to be employed for a short duration and can be supplemented or even substituted with free options. Jukensei, however, are likely to have attended cram schools dedicated to examination preparation for years prior to taking the juken. Combining the tuition of these cram schools with the fees charged to take mock examinations, the Center Test, and university specific examinations, the cost to be well-prepared and go through with the juken a single time defies comparison to that of taking that SAT even multiple times. Many jukensei go on to become roninsei-a student who does not enter university on his or her first attempt and spends a minimum of one year preparing and retrying. For the parents of roninsei, costs are often more than doubled as it typically means a full-time juku schedule. It is understandable, then, that jukensei express a sense of obligation and gratitude towards their parents.

[Juken] Both of my parents have spent a lot of money to support me. They're my parents and I'm their child so they're supporting me. But, I see myself beginning to think that this is just natural. I take lots of examinations and each one costs money. The winter semester will be even more expensive. I want to be able to pay them back for these things. That thought is present in my mind every day. [Support-Family; Progress-Goals]

Such expressions of obligation and gratitude are not only aimed at parents or limited in scope to financial support.

[Juken] I know I need to stay dedicated, but doing it is just so tough. There are so many people who have sent me heartfelt messages saying they're hoping I'll pass. I even got handwritten letters from some people. It makes me very happy. I want to meet their expectations [...] You mustn't betray people's hopes for you. I will show them their hopes were well placed. [Valence-Positive/Negative; Support; Progress-Goals] 
[Juken] When I think about the people who are rooting for me, I deeply feel that I no longer have the option of retreating. [Support]

The sense of obligation and gratitude expressed here is of a different sort. The posters express feelings of gratitude and an owing of something to others, but they suggest that, should they be unable to repay what is owed, it would constitute a betrayal rather than a mere failure-it would have implications for their character rather than just for their capabilities.

Other social media users were also identified in expressions of gratitude. Posters spoke about relying on the support they received through the platforms, and about struggling to communicate with others who were not engaged in juken preparation. There were also, however, times when jukensei communicated that they needed to withdraw from social media for extended periods because they felt the need to focus entirely on examination preparation.

Jukensei also regularly identified the avoidance of future regrets as a source of motivation.

Similar sentiments were not communicated by the SAT students.

[Juken] Whether I laugh or cry, I have 22 days. I have to work hard until the end. The only thing I do not want is to have regrets. [Progress-Goals; Examinations-Time left before]

\section{Discussion}

This section will largely seek to address the third question identified in the introduction of this paper, that being: How closely aligned are the structural-functional elements of the examinations and the intra-group communications they produce and what does this suggest about the experience of preparing for either?

Among the most apparent answers to this question is that both groups communicated their displeasure with the process. Posts with negative valence outnumbered those with positive by two to one for jukensei and by nearly three to one for the SAT group. While unsurprising, this imbalance cannot be entirely attributed to the nature of the process given that one of the most robust findings from social psychology and behavioral economics is the general human bias towards negativity (Hanson, 2014; Vaish, Grossmann and Woodward, 2008; Rozin and Royzman, 2001; Taylor, 1991). This, however, only further supports the likelihood that the process is largely experienced as unpleasant.

It is noteworthy that jukensei expressed a lower ratio of negative sentiments despite the objectively more demanding process in which they are engaged. Part of the explanation for this almost certainly lies in differences between the schooling and socialization undergone by the two groups. But, the possibility that jukensei communicate negative sentiments to one another at a lower rate precisely because they are collectively faced with a more difficult challenge must also be considered. They tended to offer advice and expressions of support in a way the SAT group did not. Perhaps, then, they perceive themselves as accountable for how they individually shape the collective experience of struggle in which they are engaged.

This is in keeping with the fundamentally instrumental, 'in order to' orientation of Western cultures and, in contrast, with the obligatory, 'because' motivations that characterize Japanese cultural pscyhology (Davis, 1992). Rather than harboring a desire to change the world to suit them, jukensei express more thorough acceptance of their need to change themselves in response to what is.

Combined with the unpleasantness of preparing, the prolonged nature of the process inspired both groups to express feelings of mounting anxiety and to speak longingly of a time when the examinations would be over. This combination of unpleasantness and prolonged-ness also likely explains the support-focused communications of both groups. This support was variably portrayed as coming from food and drink, friends, teachers, family members, faith, and social media followers, but its necessity was consistently communicated. 
The juken produced more intense support-focused posts, and a greater variety of them. This can clearly be connected to the major ways in which the juken differs structurally from the SAT: its greater consequence, its lower frequency, and its higher costs. Jukensei expressed more of a need for support, more appreciation for the support they received, and more concern over how they could fulfill the obligations entailed by that support. They communicated these messages both with their words and with the nature of their social media use.

One example of the latter is how jukensei not only engaged with social media at a rate dwarfing that of the SAT group but, with rare exception, did so anonymously. It might be suggested that this is merely how macro-level cultural differences (e.g. the tailoring of self-presentation according to the inner- or outer-ness of the audience) manifests vis á vis social media usage, but it is hardly difficult to find Japanese posters elsewhere on the services who readily show their faces. Jukensei as a group, however, are not so disposed. If their communications are taken as genuine, and given the content of those communications there appears little reason not to do so, they appear to use social media in an anonymously intimate manner seemingly motivated to divulge the details of their experiences in an authentic, vulnerable way while deigning to share much related to their identities. They post exhaustive mock examination score reports in their entirety. They talk about grudges they are harboring, moments of tragedy and despair they have experienced, and issues of mental illness they have kept secret even from their closest family members. And the networks they tend to form through social media, while more often composed of strangers than those of SAT students, appear to be no less reliable and far more task-oriented sources of support. Whereas in most contexts anonymity facilitates anti-social behaviors by undermining the human connection (Zimbardo, 2007), in this case it may be their anonymity acting in concert with the knowledge that the anonymous other is struggling with similar difficulties that allows jukensei to feel free enough to seek and be amenable to authentic connections.

The comparatively higher rate of support-focused posts also aligns with the higher economic burden imposed by the juken. This burden is severe enough to make practically infeasible the notion of a school-aged individual managing it without some sort of support network. The financial costs are high even considering only the cost of mock and real examinations. But the costs in time impose no less of a demand, making the everyday support activities performed by families-such as food preparation-much more valuable. The jukensei posts suggest a daily schedule arranged around eight to ten hours of studying, and this is the same range reported in interviews with former roninsei (Roth, 2019). Thus, straight lines can be drawn from the financial and temporal costs of juken preparation to the expressions of gratitude and indebtedness posted by jukensei.

The standardized nature of the examinations aligns well with the focus on results evidenced in both groups by the common practice of reporting and discussing scores. Proper preparation for examinations such as these affords little time to engage in magical thinking-answers are right or wrong and, if one intends to do well, the results of pre-tests can only be taken as accurate measures of one's current ability. While the score-focused posts made by the SAT group only addressed the poster's own performance, the jukensei often portrayed higher achievement as zero-sum, casting jukensei who were not their friends or supporters, and sometimes even these, as rivals. This is likely evidence of how several structural factors coalesce. The first, discussed earlier, is the Mt. Fujirather-than-market character of higher education in Japan as it encourages students to participate in a king-of-the-hill (or mountain) dynamic rather than to seek institutions of best fit. The second is the mock examination system which provides jukensei, throughout the course of the preparation process, with ordinal representations of their success. And, whereas it is easy to believe that SAT small score differentials can be offset by other factors, the weightiness of the juken means that jukensei can succeed or fail based on a single incorrect answer. Each of these factors contributes to juken preparation being collectively represented as a competition in ways that SAT preparation is not. While awareness of the competitive nature of the process may have existed on the periphery for SAT group, among jukensei it treated as a centerpiece. 
If juken preparation is a contest, it is most certainly a race run against one's peers and the clock. The passing of time was a consistent theme in the communications produced by jukensei. The SAT group never expressed concerns over remaining preparation time, and this suggests that the frequency with which the SAT is offered effectively removes this concern from the intra-group conservation. The jukensei, posted about time with near obsession-whether they had spent enough of it studying, whether enough of it remained, and how to best make use of what there was. Having only one chance a year at the examination seems, for this group, to have paradoxically cast time as both treasured friend and feared enemy.

And this brings the discussion to the seriousness disparity apparent between the data sets. As opposed to the language of inconvenience used by the SAT group, the dire language used by jukensei suggests that the more determinative function and farther-reaching consequences of the juken produce more and stronger expressions of concern, giving the conversation at times either a more hysteric or resigned quality. The SAT group brought up the examination during the course of what appeared normal banter. Given what loomed in their collective future, the jukensei struggled to engage in normal banter and, in some cases, foreswore it altogether.

\section{Limitations}

This study is limited in the nature of the data it employed. While the quantity of data dealt with in this paper-well over 10,000 lines of text and approximately 1,000 images-is certainly large enough to have conducted a robust thematic analysis, other data sources would be expected to augment the findings here presented with additional layers of complexity.

It must also be acknowledged that inequality plays a role in the experience and outcomes of these examinations. While analysis of the data uncovered themes of economic anxiety and difficulty, the study was unable to comment in a deep way on how various levels of inequality affect the posting behavior. The fact that economic insecurity prevents many from engaging with these examinations at all must also be acknowledged.

Other limitations include the study failing to address elements of engagement such as likes and replies that might have elucidated the nature and range of effects associated with these posts.

\section{Conclusion}

While the reliability of social media posts as a window onto the genuine experiences of populations such as these has not yet been firmly established, their posting habits undoubtedly serve some perceived need. They also describe, highlight, and reflect aspects of a shared experience and, thereby, contribute to the formation of a collective one. Given the conspicuous structural and functional similarities of the examinations in question-their consequence, lengthiness, standardization, standing outside of compulsory curriculum, and roles as gatekeepers for tertiary education-the shared public expressions of these groups are as expected. They generally claim to be unhappy with the demands imposed by these examinations, but they also communicate the importance of industriousness and a results-based appraisal of their abilities. Given the structural and functional differences between these examinations-frequent as opposed to yearly, relatively shorter and less preparation intensive as opposed to longer and more intensive, relatively cheap as opposed to dependency-inducing, and one element as opposed to the element-the intra-group communications they produce are perhaps less predictable. Jukensei post more about being engaged in a process of self-overcoming. They seek and provide more support, often by interacting anonymously with individuals they only know through social media. They post more and much more frequently. They openly address the competitive aspects of the preparation process, cultivating supportive rivalries. They are more likely to describe their upcoming examination in dire terms and to express feelings of gratitude and indebtedness directed at those supporting their preparation efforts. 
These examinations exhibit sufficient structural and functional similarity to make them convenient points of comparison. As tests in the broader sense, however, they are unique manifestations of the socio-cultural systems in which they are co-determinatively embedded, and of the individual stories and collective character of the populations that undergo them.

\section{Notes}

${ }^{1}$ roninsei is a student who does not enter university on his or her first attempt and spends a minimum of one year preparing and retrying

\section{References}

Arai, A. G.(2016). The Strange Child: Education and the Psychology of Patriotism in Recessionary Japan. Stanford: Stanford University Press.

Aspinall, R.(2003). Japanese nationalism and the reform of English language teaching. In R. Goodman and D. Phillips (Eds.) Can the Japanese Change their Education System? Oxford: Symposium Books, pp. 103-118.

Aspinall, R.(2005) University entrance in Japan. In J.S. Eades, R. Goodman, and Y. Hada (Eds.) The 'Big Bang' in Japanese Higher Education: The 2004 Reforms and the Dynamics of Change. Melbourne: Trans Pacific Press, pp. 199-218.

Banathy, B.(1995). Developing a systems view of education. Educational Technology, 35(3), pp. 53-57. Brinton, M. C.(1993). Women and the Economic Miracle. Berkeley: The University of California Press. CollegeBoard(2016). One year since launch, official SAT practice on Khan Academy is leveling the playing field for students. Available at: https://www.collegeboard.org/releases/2016/One-YearSinceLaunch-SAT-Khan-Academy-Leveling-Playing-Field [Accessed 7 March 2019].

CollegeBoard (2019) Test dates and deadlines. Available at: https://collegereadiness.collegeboard. org/sat/register/dates-deadlines [Accessed 4 September 2019].

Compare SAT Specifications (n.d.). CollegeBoard. Available at https://collegereadiness.collegeboard. org/sat/inside-the-test/compare-old-new-specifications [Accessed 5 March 2019].

Daigaku Nyushi Senta[Center Exam for University Entrance] (NCUEE) (2015). NCUEE. Available at: https://www.dnc.ac.jp/albums/abm00006725.pdf [Accessed 20 March 2019].

Davis, W.(2019). Japanese Religion and Society: Paradigms of Structure and Change. Albany: State University of New York Press.

De Vos, G. A.(1973). Socialization for Achievement. Berkeley: University of California Press.

FairTest(2019). More than 1000 accredited colleges and universities that do not use ACT/SAT scores to admit substantial numbers of students into bachelor-degree programs. Available at: http:// www.fairtest.org/university/optional [Accessed 5 March 2019].

Foreign Press Center Japan (FPCJ) (2018). Education and entrance exam reforms. Available at: https:// fpcj.jp/en/j_views-en/magazine_articles-en/p=62839/[Accessed 3 March 2019].

Goodman, R.(2003). The why, what and how of educational reform in Japan. In R. Goodman and D. Phillips (Eds.) Can the Japanese Change their Education System? Oxford: Symposium Books, pp. 7-30.

Guest, M.(2006). Teaching progressively... for the senta shiken! In K. Bradford-Watts, C. Ikeguchi, and M. Swanson (Eds.) JALT 2005 Conference Proceedings. Tokyo: JALT, pp. 1182-1191.

Hanson, R.(2014). Taking in the good vs. the negativity bias. Available at: https://www.sfsu. edu/ holistic/documents/Spring_2014/GoodvsNeg_Bias.pdf [Accessed 11 March 2019].

Hofstede, G., Hofstede, G. J., and Minkov, M.(2010). Cultures and Organizations: Software of the Mind. New York: McGraw-Hill.

Hogue, J. V. and Mills, J. S.(2018). The effects of active social media engagement with peers on body image in young women. Body Image, 28, pp. 1-5. 
Kawaijuku(2019). Juken-ryō(Examination Fee). Available at https://www.kawai-juku.ac.jp/trial-exam/ zento/fee/[Accessed 25 August 2019].

Kinmouth, E.(2005). From selection to seduction: The impact of demographic change on private higher education in Japan. In J. S. Eades, R. Goodman, and Y. Hada (Eds.). The 'Big Bang' in Japanese Higher Education: The 2004 Reforms and the Dynamics of Change. Melbourne: Trans Pacific Press, pp. 106-136.

McCrostie, J. (2017). Spoken English tests among entrance exam reforms Japan's students will face in 2020. The Japan Times. Available at https://www.japantimes.co.jp/community/2017/07/05/ issues/spoken-english-tests-among-entrance-exam-reforms-japans-students-will-face-2020/ [Accessed 6 March 2019].

McNeill, D.(2008). Facing enrollment crisis, Japanese universities fight to attract students. The Chronicle of Higher Education. Available at http://www.chronicle.com/article/FacingEnrollment-Crisis/6649[Accessed 4 March 2019].

McVeigh, B. (2000). Wearing Ideology: State, Schooling and Self-Presentation in Japan. Oxford: Berg. Meyer, E.(2014). The Culture Map. New York: PublicAffairs.

Montoya, J. and Camara, W.(2012). SAT report on college and career readiness. Available at http:// media.collegeboard.com/homeOrg/content/pdf/SAT\%20Report_Press\%20Conferece\%20 Powerpoint\%20_FINAL\%20with\%20NOTES.pdf [Accessed 23 April 2019].

Mori, R.(2002). Entrance examinations and remedial education in Japanese higher education. Higher Education, 43(1), pp. 27-42.

Mulvey, B.(2001). The role and influence of Japan's university entrance examinations: A reassessment. The Language Teacher. Available at: http://jalt-publications.org/old_tlt/articles/2001/07/ mulvey[Accessed 28 February 2019].

Murphy, R. T.(2014). Japan and the Shackles of the Past. Oxford: Oxford University Press.

Ozawa-de Silva, C.(2008). Too lonely to die alone: Internet suicide pacts and existential suffering in Japan. Culture, Medicine and Psychiatry, 32(4), pp.516-551.

Puglia, J.(2016). Going away to college is a rite of passage. Los Angeles Times. Available at: https:// www.latimes.com/socal/la-canada-valley-sun/opinion/tsn-vsl-thoughts-from-drjoe-goingaway-to-college-is-a-rite-of-passage-20160818-story.html[Accessed 8 March 2019].

Rohlen, T.(1983). Japan's High Schools. Berkeley: University of California Press.

Roth, I.(2019). Chûshingura: The roninsei experience in contemporary Japan. Proquest Open.

Rozin, P. and Royzman, E. B.(2001). Negativity bias, negativity dominance, and contagion. Personality and Social Psychology Review, 5(4), pp. 296-320.

Sakamoto, A. and Powers, D.(1995). Education and the dual labor market for Japanese men. American Sociological Review, 60(2), pp. 222-246.

Shanahan, N., Brennan, C. and House, A. (2019). Self-harm and social media: Thematic analysis of images posted on three social media sites. BMJ Open 2019(9). doi: 10.1136/ bmjopen-2018-027006

Sheftall, M. G.(2011). Shattered gods: The unresolved cultural consequences of Japan's post-1945 desymbolization crisis. Presented at the Association of Asian Studies Convention. Available at: www.debito.org/?p=9147[Accessed 3 March 2019].

Social Media Research Group. (2016). Using social media for social research: An introduction. Social Science in Government. Available at: https://assets.publishing.service.gov.uk/government/ uploads/system/uploads/attachment_data/file/524750/GSR_Social_Media_Research_ Guidance_-_Using_social_media_for_social_research.pdf [Accessed 16 August 2019].

Takeuchi, Y.(1997). The self-activating entrance examination system-its hidden agenda and its correspondence with the Japanese "salary man". Higher Education, 34(2), pp.183-198.

Taylor, S. E.(1991). Asymmetrical effects of positive and negative events: The mobilization minimization hypothesis. Psychological Bulletin, 110(1), pp.67-85.

Tsuda, T.(1993). The psychosocial functions of liminality: The Japanese university experience. The Journal of Psychohistory, 20(3), pp. 305-330. 
Tsukada, M.(1991). Yobiko Life: A Study of the Legitimation Process of Social Stratification in Japan. Berkeley: University of California Press.

Turkle, S.(2011). Alone Together. New York: Basic Books.

Vaish, A., Grossman, T. and Woodward, A.(2008). Not all emotions are created equal: The negativity bias in social-emotional development. Psychological Bulletin, 134(3), pp. 383-403.

Vieira, K. A. and de Queiroz, G. M. (2017). Hermeneutic content analysis: A method of textual analysis. International Journal of Business Marketing and Management, 2(8), pp. 8-15.

Yamamoto, B. A., Ishikura, Y. and Saito, T.(2016). University recognition of diploma programme graduates at leading Japanese universities. IBO. Available at https://www.ibo.org/ contentassets/d1c0accb5b804676ae9e782b78c8bc1c/research-dp-japan-universityrecognition-en.pdf[Accessed 11 March 2019].

Zeng, K.(1995). Japan's dragon gate: The effects of university entrance examinations on the educational system and students. Compare: A Journal of Comparative Education, 25(1), pp. 59-84.

Zeng, K.(1999). Dragon Gate: Competitive Examinations and their Consequences. London: Cassell. Zimbardo, P.(2007). The Lucifer Effect. New York: Random House. 


\section{Appendix A}

Jukensei Coding Results

\begin{tabular}{|c|c|c|}
\hline Theme & Appearances & Rate of Appearance in Sections \\
\hline Being jukensei & 141 & $100 \%$ \\
\hline -Advice about & 42 & $61.5 \%$ \\
\hline -Characterizations & 61 & $96.2 \%$ \\
\hline -Experience of time & 34 & $72 \%$ \\
\hline -Number of years & 4 & $11.5 \%$ \\
\hline Condition & 120 & $84.6 \%$ \\
\hline -Environmental & 34 & $73.1 \%$ \\
\hline -Mental & 29 & $46.2 \%$ \\
\hline -Physical & 57 & $76.9 \%$ \\
\hline Effort & 272 & $100 \%$ \\
\hline -Intention & 222 & $100 \%$ \\
\hline -Lack & 25 & $53.8 \%$ \\
\hline -Prior & 25 & $53.8 \%$ \\
\hline Examinations & 379 & $100 \%$ \\
\hline -Center & 26 & $57.7 \%$ \\
\hline -Post- & 14 & $34.6 \%$ \\
\hline -Practice and Pre & 156 & $100 \%$ \\
\hline -Time left before & 170 & $100 \%$ \\
\hline -University specific & 13 & $30.8 \%$ \\
\hline Food and Drink & 79 & $76.9 \%$ \\
\hline Leisure & 32 & $50 \%$ \\
\hline Part-time Job & 17 & $38.5 \%$ \\
\hline Preparation & 723 & $100 \%$ \\
\hline -Plans & 155 & $96.2 \%$ \\
\hline -Religious & 18 & $42.3 \%$ \\
\hline -Routines and Systems & 19 & $50 \%$ \\
\hline -School and Home & 42 & $80.8 \%$ \\
\hline -Self & 6 & $15.4 \%$ \\
\hline -Time studying & 117 & $92.3 \%$ \\
\hline -Materials & 366 & $100 \%$ \\
\hline Progress & 427 & $100 \%$ \\
\hline -Ability and Difficulty & 124 & $100 \%$ \\
\hline -Goals & 107 & $96.2 \%$ \\
\hline -Motivation & 62 & $69.2 \%$ \\
\hline -Position & 20 & $42.3 \%$ \\
\hline
\end{tabular}




\begin{tabular}{|l|l|l|}
\hline -Results & 114 & $92.3 \%$ \\
\hline Reflection & 57 & $88.5 \%$ \\
\hline -Earlier this year & 5 & $11.5 \%$ \\
\hline -Last year or earlier & 52 & $88.5 \%$ \\
\hline Support & 239 & $100 \%$ \\
\hline -Family & 48 & $84.6 \%$ \\
\hline -Followers (SNS) & 83 & $92.3 \%$ \\
\hline --SNS use & 29 & $65.4 \%$ \\
\hline -Friends & 27 & $84.6 \%$ \\
\hline -Teachers & 54 & $80.8 \%$ \\
\hline University & 71 & $80.8 \%$ \\
\hline -Life & 11 & $26.9 \%$ \\
\hline -Of interest & 60 & $76.9 \%$ \\
\hline Valence & 145 & $100 \%$ \\
\hline -Negative & 95 & $100 \%$ \\
\hline -Positive & 50 & $84.6 \%$ \\
\hline
\end{tabular}

\section{Appendix B}

SAT Group Coding Results

\begin{tabular}{|l|l|l|}
\hline Theme & Total Appearances & Rate of Appearance \\
\hline Valence & 45 & $67.2 \%$ \\
\hline -Positive & 13 & $19.4 \%$ \\
\hline -Negative & 32 & $47.8 \%$ \\
\hline Complaint & 17 & $25.4 \%$ \\
\hline Schedule & 10 & $14.9 \%$ \\
\hline Ability & 10 & $14.9 \%$ \\
\hline Support & 13 & $19.4 \%$ \\
\hline Materials & 10 & $14.9 \%$ \\
\hline Effort & 8 & $11.9 \%$ \\
\hline -Intended & 2 & $3.0 \%$ \\
\hline -Prior & 6 & $8.9 \%$ \\
\hline Procrastination & 5 & $7.5 \%$ \\
\hline Anxiety & 4 & $6.0 \%$ \\
\hline Food/Drink & 6 & $8.9 \%$ \\
\hline College & 1 & $1.5 \%$ \\
\hline Religion & 3 & $4.5 \%$ \\
\hline Break & 5 & $7.5 \%$ \\
\hline Goal & 1 & $1.5 \%$ \\
\hline & & \\
\hline
\end{tabular}


